\title{
Residual Effect of Applied Vermicompost and NPK to Rice on Growth and Yield of Succeeding Wheat and Chemical Properties of Soil
}

\author{
Amit Kumar ${ }^{1}$, B.P. Dhyani ${ }^{1}$, Ashish Rai ${ }^{2 *}$ and Vipin Kumar ${ }^{2}$ \\ ${ }^{1}$ Department of Soil Science, Sardar Vallabhbhai Patel University of Agriculture and \\ Technology, Meerut, Uttar Pradesh, India \\ ${ }^{2}$ Department of Soil Science and Agricultural Chemistry, Institute of Agricultural Sciences, \\ Banaras Hindu University, Varanasi, Uttar Pradesh, India \\ *Corresponding author
}

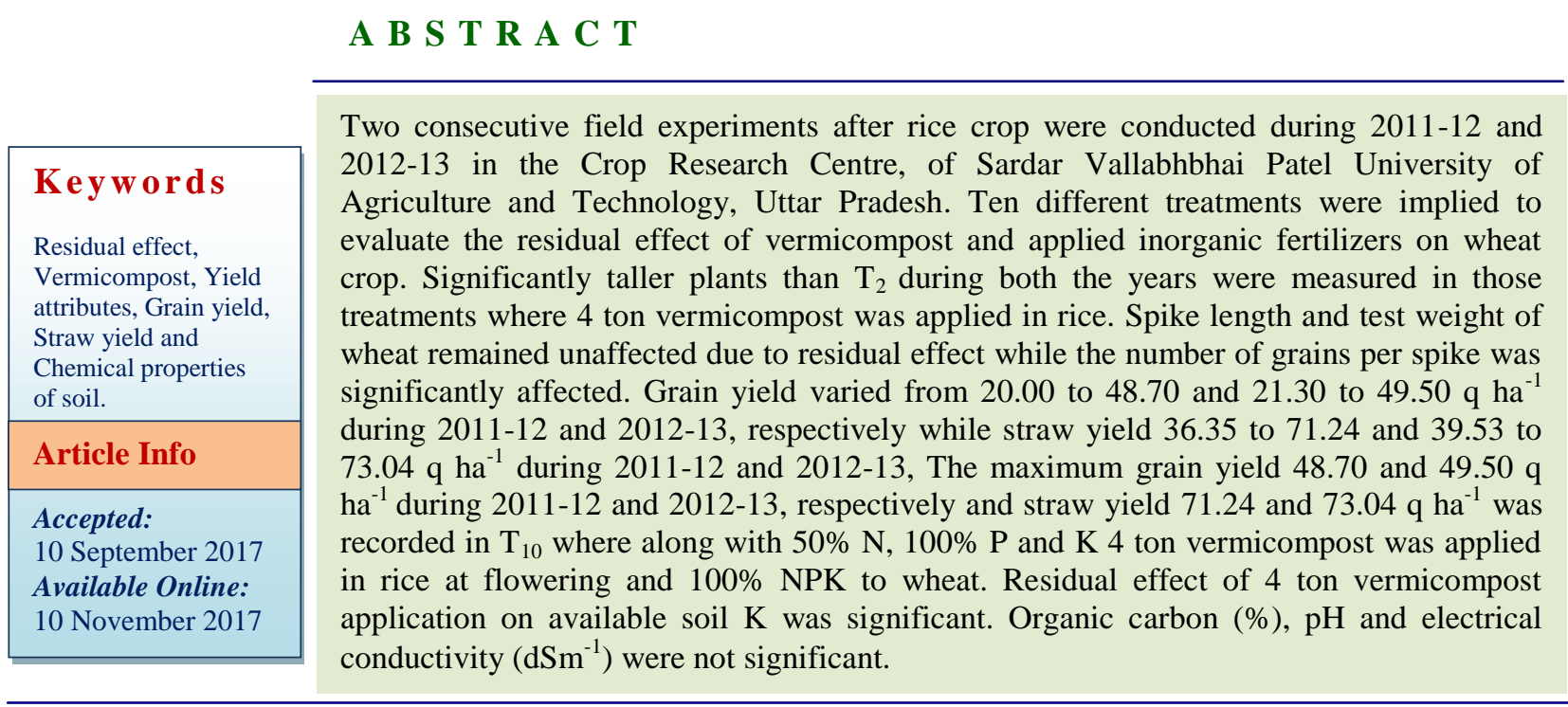

\section{Introduction}

From the time of independence in 1947 until 1965, agricultural production in India was unable to meet the country's needs. Severe droughts in the mid-1960s threatened famine which was averted only by substantial shipments of food grains from the United States. The green revolution i.e. introduction of the new high yielding varieties of wheat, rice and maize was the technological response to a worldwide food storage which became threatening in the period after World War II. The green revolution transformed farming practice in many regions of the tropics and sub tropics where the principal food crops were rice, wheat and maize. The average wheat productivity of India is $2.83 \mathrm{t} \mathrm{ha}^{-1}$. No doubt increased production of rice and wheat filled self-sufficiency in food grain production, however, indiscriminate use of inorganic fertilizers and plant protection chemicals for maximizing crop yield has resulted in the deterioration of the physical, chemical and biological properties of ricewheat growing soils. There is a growing 
concern about the sustainability of the ricewheat cropping system as the growth rate of rice and wheat yields are either stagnant or have declined. Therefore despite the early benefits, it became apparent that there are many negative impacts from the green revolution such as reduced natural fertility of soil, salinization of the agricultural soil, decline in soil organic matter, and poor soil physical condition particularly in rice-wheat system.

To sustain or increase the productivity of ricewheat system, it is important that soil status must be perfect the level of organic matter in soil should be enough and overall the soil must be without any constraints. Some of the secretions of worms and associated microbes act as growth promoter along with other nutrients. It has attracted the attention not only of scientists but also of farmers worldwide. Since it is a natural organic product which is eco-friendly, it does not leave any adverse effects either in the soil or in the environment. The $\mathrm{C} / \mathrm{N}$ ratio of vermicompost is much lower $(16: 1)$ than that of FYM (30:1). The effect of earthworms on plant growth may be due to several reasons apart from the presence of macro and micronutrients in their secretions and vermicast in considerable quantities. Certain metabolites produced by earthworms may also be responsible to stimulate the plant growth. Vermicompost also helps in preventing plant diseases (Rao, 2000). The mucus associated with the cast being hygroscopic absorbs water and improve water holding capacity. A positive effect of vermicompost application on yield attributes and yield of various crops had been reported by Vasanthi and Kumar Swamy (2000), Ranwa and Singh (1999), Das et al., (2002) and Singh et al., (2005). Dussere (1992) reported that vermicompost helps to improve and protect fertility of top soil and also helps to boost up productivity by $40 \%$ with 20 to
$60 \%$ lower inputs, It also enhance the quality of end products and thereby creating significant impact on flexibility in marketing as well as increases the storage time. Vermicompost contain 30 to 50 percent substance which help in the stimulation of plant growth, particularly that of roots. Robinson et al., (1992) reported that the nutrients present in vermicompost are readily available. In the present study an attempt was made to study the effect of timing of vermicompost application in integrated mode on main crop rice and its residual effect on succeeding wheat crop.

\section{Materials and Methods}

Two field experiments were conducted at the Crop Research Centre (CRC), Chirodi of Sardar Vallabhbhai Patel University of Agriculture and Technology, Meerut (U.P.) located at latitude of $29^{\circ} 40^{\prime}$ north and longitude of $77^{\circ} 42^{\prime}$ east and at an altitude of 237 meter above mean sea level (MSL). The area receives on an average $862 \mathrm{~mm}$ of rains annually of which $90 \%$ is confined to rainy season.

A composite soil sample was collected from a depth of $0-15 \mathrm{~cm}$ of the experimental site. The experimental soil was sandy loam in texture (Sand, 63.2\%, Silt $18.5 \%$ and Clay $18.3 \%$ ), alkaline in $\mathrm{pH}$ (8.04), having low organic carbon $(0.43 \%)$, available nitrogen (155.5 $\mathrm{kg} / \mathrm{ha})$ medium in phosphorus (13.8 kg/ha) and potassium $(141.5 \mathrm{~kg} / \mathrm{ha})$. To study the residual effect of vermicompost on growth performance of wheat crop and chemical properties of soil ten treatments were implied in randomized block design with three replication in rice-wheat system during 201112 and 2012-13. Treatment details included, $\mathrm{T}_{1}$ - Control without NPK in rice and wheat, $\mathrm{T}_{2}-100 \%$ RDF to rice and wheat, $\mathrm{T}_{3}-75 \%$ $\mathrm{N}, 100 \%$ P\&K + Vermicompost @ $2 \mathrm{t} \mathrm{ha}^{-1}$ as basal to rice followed by $100 \%$ RDF to wheat, 
$\mathrm{T}_{4}-75 \% \mathrm{~N}, 100 \% \mathrm{P} \& \mathrm{~K}+$ Vermicompost @ 2 ton $\mathrm{ha}^{-1}$ at tillering stage to rice followed by $100 \%$ RDF to wheat, $\mathrm{T}_{5}-75 \% \mathrm{~N}, 100 \% \mathrm{P} \& \mathrm{~K}$ + Vermicompost @ 2 ton ha ${ }^{-1}$ at panicle initiation followed by $100 \%$ RDF to wheat, $\mathrm{T}_{6}$ $-75 \% \mathrm{~N}, 100 \% \mathrm{P} \& \mathrm{~K}+$ Vermicompost @2 ton $\mathrm{ha}^{-1}$ at flowering stage to rice followed by $100 \%$ RDF to wheat, $\mathrm{T}_{7}-50 \% \mathrm{~N}, 100 \% \mathrm{P} \& \mathrm{~K}$ +Vermicompost @ 4 ton $\mathrm{ha}^{-1}$ as basal to rice followed by $100 \%$ RDF to wheat, $\mathrm{T}_{8^{-}}-50 \%$ $\mathrm{N}, 100 \%$ P\&K + Vermicompost @ 4 ton ha ${ }^{-1}$ at tillering stage to rice followed by $100 \%$ RDF to wheat, $\mathrm{T}_{9}-50 \% \quad \mathrm{~N}, 100 \%$ P\&K + Vermicompost @ 4 ton $\mathrm{ha}^{-1}$ at panicle initiation to rice followed by $100 \% \mathrm{RDF}$ to wheat and $\mathrm{T}_{10} \quad-50 \% \quad \mathrm{~N}, 100 \% \quad \mathrm{P} \& \mathrm{~K} \quad+$ Vermicompost @ 4 ton ha $^{-1}$ at flowering stage to rice followed by $100 \% \mathrm{RDF}$ to rice. RDF for rice 120, 60, $60 \mathrm{~N} \mathrm{P}$ and $\mathrm{K} \mathrm{kg} \mathrm{ha}^{-1}$ and for wheat $150,75,60 \mathrm{~kg} \mathrm{ha}^{-1}$ for both the years.

\section{Observations recorded during crop growth and after harvest}

Growth observations viz. number of tillers per meter length from the randomly selected row at harvesting, plant height was measured with the help of meter scale from the base of plant to the tip of the tallest leaf up to emergence of spike and thereafter up to the tip of panicle/ spike average shoot height was computed and expressed in $\mathrm{cm}$. at harvest, Length of five panicles or spikes were measured, Number of grains obtained from the five panicles or spikes were counted and expressed as mean number of grains per panicle or spike, 1000 grains were counted and weighed for test weight which was expressed in gram, the weight of grains of wheat harvested from the net plot area was recorded in $\mathrm{kg}$ and finally expressed in $\mathrm{q} \mathrm{ha}^{-1}$.

The straw yield was computed on difference basis. Grain yield was subtracted from the biological yield of net plot and expressed as $\mathrm{kg} \mathrm{ha}^{-1}$ and finally expressed in $\mathrm{q} \mathrm{ha}^{-1}$.

\section{Chemical analysis}

The soil samples were collected after harvesting of the wheat and processed with standard procedure. The $\mathrm{pH}$ was determined in soil water suspension $(1: 2)$ was determined with the help of glass electrode on a $\mathrm{pH}$ meter (Jackson, 1967). The soluble salts $\left(\mathrm{dSm}^{-1}\right)$ in soils were measured with a conductivity meter, also known as "salt bridge". Organic carbon was estimated by wet digestion method as described by Jackson (1967). Available $\mathrm{N}$ was determined by alkaline permanganate method. The determination of available phosphorus was done by Olsen's method (Olsen et al., 1954). Available potassium was determined by using neutral ammonium acetate as an extractant and potassium concentration was read by using flame photometer in the filtered extract. The raw data collected for all parameters at different crop stages during the course of investigation was compiled and subjected to statistical analysis using the analysis of variance technique (Gomez and Gomez, 1984). The critical difference (at $5 \%$ level of probability) was computed for comparing treatment mean in cases where effect came out to be significant by F- test.

\section{Results and Discussion}

\section{Tillers/m row length and plant height $(\mathrm{cm})$}

With exception of $T_{1}$, the tillers/meter row length remained unaffected due to residual effect of preceding rice crop treatments, although comparatively more number of tillers than $T_{2}$ were counted in those treatments where 4 ton vermicompost was applied to rice (Table 1). Significantly lower number of tillers/meter row length than the remaining treatments were counted in $T_{1}$. Significantly taller plants than $\mathrm{T}_{2}$ during both the years were measured in those treatments where 4 ton vermicompost was applied in rice 
at various growth stages. Delayed application of 4 ton vermicompost to rice also resulted in significantly taller plant than the treatments where early application of 2 ton vermicompost was made. The number of tillers/m row length of wheat at harvest did not differ significantly due to residual effect of applied vermicompost. Although number of tillers $/ \mathrm{m}$ row length in the treatments consisting application of 4 ton vermicompost to rice were comparatively higher than $T_{2}$ (100\% NPK) but difference could not reach to the level of significance.

\section{Spike length $(\mathrm{cm})$, number of grains per} spike and test weight (g) of wheat

Spike length $(\mathrm{cm})$ remained unaffected due to residual effect and ranged from 7.20 to 10.80 and 7.90 to $11.30 \mathrm{~cm}$ during 2011-12 and 2012-13, respectively (Table 2). The maximum spike length 10.80 and 11.30 during 2011-12 and 2012-13, respectively, was recorded in $\mathrm{T}_{10}$ where along with $50 \% \mathrm{~N}$, $100 \% \mathrm{P}$ and $\mathrm{K}, 4$ ton vermicompost was applied in rice at flowering stage and $100 \%$ NPK to wheat.

The number of grains per spike varied from 25.30 to 55.90 and 26.40 to 56.00 during 2011-12 and 2012-13, respectively (Table 2). The maximum grains per spike than the other treatments 55.90 and 56.00 during 2011-12 and 2012-13 were recorded in $\mathrm{T}_{10}$ where along with $50 \% \mathrm{~N}, 100 \% \mathrm{P}$ and $\mathrm{K}, 4$ ton vermicompost was applied in rice at flowering and $100 \%$ NPK to wheat. Only $\mathrm{T}_{1}$ was found significantly inferior to rest of the treatments in respect of number of grains per spike while remaining treatments were statistically at par.

It is clear from the table 2 that the test weight remained unaffected due to residual effect during both the years. The test weight $(\mathrm{g})$ varied from 36.48 to 39.18 and 36.74 to 39.51g during 2011-12 and 2012-13, respectively. The maximum test weight during both the years was found in $T_{10}$. Delayed application of 4 ton vermicompost in rice resulted in slightly higher test weight than the $\mathrm{T}_{2}$. Delayed application of 4 ton vermicompost to rice crop resulted in comparatively higher yield attributing characters. In case of number of grains per spike only $T_{1}$ was found significantly inferior than the rest of the treatments. Higher number of grains was found with delayed application of vermicompost to rice. This variation may be due to comparatively better physical environment that affects nutrient transformation and absorption from the soil and thereby responds to vegetative as well as reproductive phase of plants.

\section{Grain yield ( $\left.\mathbf{q} h^{-1}\right)$, Straw yield $\left(q h^{-1}\right)$ and Biomass yield}

Grain yield varied from 20.00 to 48.70 and 21.30 to $49.50 \mathrm{q} \mathrm{ha}^{-1}$ during 2011-12 and 2012-13, respectively (Table 3). The maximum grain yield 48.70 and $49.50 \mathrm{q} \mathrm{ha}^{-1}$ during 2011-12 and 2012-13, respectively, was recorded in $\mathrm{T}_{10}$ where along with $50 \% \mathrm{~N}$, $100 \% \mathrm{P}$ and $\mathrm{K}, 4$ ton vermicompost was applied in rice at flowering and $100 \%$ NPK to wheat. Significant residual effect of 4 ton vermicompost application to rice at different growth stages on grain yield of wheat was found.

The straw yield varied from 36.35 to 71.24 and 39.53 to $73.04 \mathrm{q} \mathrm{ha}^{-1}$ during 2011-12 and 2012-13, respectively (Table 3). The maximum straw yield 71.24 and $73.04 \mathrm{q} \mathrm{ha}^{-1}$ during 2011-12 and 2012-13, was recorded in $\mathrm{T}_{10}$ where along with $50 \% \mathrm{~N} 100 \% \mathrm{P}$ and K 4 ton vermicompost was applied in rice crop at flowering and $100 \%$ NPK to wheat. Residual effect of 4 ton vermicompost application to rice on straw yield of wheat was significant during both the years but no residual effect 
was obtained with the application of 2 ton vermicompost. $\mathrm{T}_{10}$ was found significantly better than $\mathrm{T}_{2}$ and all the treatments consisting application of 2 ton vermicompost to rice. Biomass yield of wheat varied from 56.35 to 119.94 and 60.83 to $122.54 \mathrm{q} \mathrm{ha}^{-1}$ during
2011-12 and 2012-13, respectively (Table 3). A significant residual effect of 4 ton vermicompost application to rice on biomass yield of wheat was observed but such effect was not noticed clearly in case of 2 ton vermicompost application.

Table.1 Residual effect of preceding rice crop treatments on plant height $(\mathrm{cm})$ and number of tillers $\mathrm{m}^{-1}$ row length of wheat crop at harvest

\begin{tabular}{|c|c|c|c|c|}
\hline \multirow{2}{*}{ Treatments } & \multicolumn{2}{|c|}{ Plant height $(\mathbf{c m})$} & \multicolumn{2}{c|}{ No. of tillers/ m row length } \\
\cline { 2 - 5 } & $\mathbf{2 0 1 1 - 1 2}$ & $\mathbf{2 0 1 2 - 1 3}$ & $\mathbf{2 0 1 1 - 1 2}$ & $\mathbf{2 0 1 2 - 1 3}$ \\
\hline $\mathrm{T}_{1}$ & 64.5 & 66.1 & 58.6 & 61.8 \\
\hline $\mathrm{T}_{2}$ & 74.4 & 76.6 & 85.8 & 86.3 \\
\hline $\mathrm{T}_{3}$ & 75.3 & 77.3 & 84.3 & 85.2 \\
\hline $\mathrm{T}_{4}$ & 76.8 & 78.2 & 86.4 & 86.7 \\
\hline $\mathrm{T}_{5}$ & 77.2 & 79.4 & 87.6 & 87.9 \\
\hline $\mathrm{T}_{6}$ & 78.6 & 81.7 & 88.5 & 87.6 \\
\hline $\mathrm{T}_{7}$ & 81.7 & 84.2 & 96.3 & 97.3 \\
\hline $\mathrm{T}_{8}$ & 83.3 & 86.1 & 94.1 & 94.5 \\
\hline $\mathrm{T}_{9}$ & 85.9 & 87.7 & 95.9 & 94.7 \\
\hline $\mathrm{T}_{10}$ & 87.1 & 88.9 & 97.3 & 95.2 \\
\hline $\mathrm{SE}(\mathrm{m})$ & 2.20 & 2.79 & 2.72 & 2.57 \\
\hline $\mathrm{CD}$ at $5 \%$ & 6.58 & 8.36 & 8.13 & 7.69 \\
\hline
\end{tabular}

Table.2 Residual effect of preceding rice crop treatments on yield attributing characters of wheat crop

\begin{tabular}{|c|c|c|c|c|c|c|}
\hline \multirow{2}{*}{ Treatments } & \multicolumn{2}{|c|}{ Length of spike(cm) } & \multicolumn{2}{c|}{ No. of grains spike } & \multicolumn{2}{c|}{ Test wt. (g) } \\
\cline { 2 - 7 } & $\mathbf{2 0 1 1 - 1 2}$ & $\mathbf{2 0 1 2 - 1 3}$ & $\mathbf{2 0 1 1 - 1 2}$ & $\mathbf{2 0 1 2 - 1 3}$ & $\mathbf{2 0 1 1 - 1 2}$ & $\mathbf{2 0 1 2 - 1 3}$ \\
\hline $\mathrm{T}_{1}$ & 7.2 & 7.9 & 25.3 & 26.4 & 36.48 & 36.74 \\
\hline $\mathrm{T}_{2}$ & 8.0 & 8.5 & 54.1 & 44.3 & 37.19 & 37.38 \\
\hline $\mathrm{T}_{3}$ & 8.2 & 8.7 & 54.3 & 54.5 & 37.45 & 37.62 \\
\hline $\mathrm{T}_{4}$ & 8.3 & 8.6 & 54.4 & 54.6 & 37.48 & 37.57 \\
\hline $\mathrm{T}_{5}$ & 8.5 & 9.2 & 54.2 & 54.4 & 37.40 & 37.52 \\
\hline $\mathrm{T}_{6}$ & 8.7 & 9.4 & 54.5 & 54.8 & 37.49 & 37.56 \\
\hline $\mathrm{T}_{7}$ & 9.5 & 10.3 & 55.6 & 55.7 & 38.37 & 38.54 \\
\hline $\mathrm{T}_{8}$ & 9.6 & 10.2 & 55.7 & 55.8 & 38.56 & 38.73 \\
\hline $\mathrm{T}_{9}$ & 9.3 & 10.6 & 55.8 & 55.9 & 38.42 & 38.95 \\
\hline $\mathrm{T}_{10}$ & 10.8 & 11.3 & 55.9 & 56.0 & 39.18 & 39.51 \\
\hline $\mathrm{SE}(\mathrm{m})$ & 2.18 & 2.10 & 2.00 & 1.86 & 2.05 & 1.79 \\
\hline $\mathrm{CD}$ at 5\% & N.S. & N.S. & 5.99 & 5.56 & N.S. & N.S. \\
\hline
\end{tabular}


Table.3 Residual effect of preceding rice crop treatments on grain, straw and biological yield $\left(\mathrm{q} \mathrm{ha}^{-1}\right)$ of wheat

\begin{tabular}{|c|c|c|c|c|c|c|}
\hline \multirow{2}{*}{ Treatments } & \multicolumn{2}{|c|}{ Grain Yield $\left(\mathbf{q} \mathbf{h a}^{-\mathbf{1}}\right)$} & \multicolumn{2}{c|}{ Straw Yield $\left(\mathbf{q} \mathbf{h a}^{-\mathbf{1}}\right)$} & \multicolumn{2}{c|}{ Biological Yield $\left(\mathbf{q ~ h a}^{-1}\right)$} \\
\cline { 2 - 7 } & $\mathbf{2 0 1 1 - 1 2}$ & $\mathbf{2 0 1 2 - 1 3}$ & $\mathbf{2 0 1 1 - 1 2}$ & $\mathbf{2 0 1 2 - 1 3}$ & $\mathbf{2 0 1 1 - 1 2}$ & $\mathbf{2 0 1 2 - 1 3}$ \\
\hline $\mathrm{T}_{1}$ & 20.0 & 21.3 & 36.35 & 39.53 & 56.35 & 60.83 \\
\hline $\mathrm{T}_{2}$ & 35.0 & 37.8 & 50.16 & 53.37 & 85.16 & 91.17 \\
\hline $\mathrm{T}_{3}$ & 37.0 & 39.2 & 51.79 & 54.29 & 88.79 & 93.49 \\
\hline $\mathrm{T}_{4}$ & 38.3 & 40.4 & 53.86 & 55.82 & 92.16 & 96.22 \\
\hline $\mathrm{T}_{5}$ & 40.0 & 43.1 & 56.52 & 58.09 & 96.52 & 101.19 \\
\hline $\mathrm{T}_{6}$ & 41.3 & 44.6 & 57.43 & 59.64 & 98.73 & 104.24 \\
\hline $\mathrm{T}_{7}$ & 43.0 & 45.8 & 61.48 & 63.75 & 104.48 & 109.55 \\
\hline $\mathrm{T}_{8}$ & 44.3 & 46.3 & 63.22 & 67.12 & 107.52 & 113.42 \\
\hline $\mathrm{T}_{9}$ & 45.3 & 47.1 & 63.47 & 62.81 & 108.77 & 109.91 \\
\hline $\mathrm{T}_{10}$ & 48.7 & 49.5 & 71.24 & 73.04 & 119.94 & 122.54 \\
\hline $\mathrm{SE}(\mathrm{m})$ & 1.97 & 1.25 & 3.36 & 3.54 & 3.25 & 3.48 \\
\hline $\mathrm{CD}$ at $5 \%$ & 5.90 & 5.22 & 10.07 & 10.61 & 9.72 & 10.41 \\
\hline
\end{tabular}

Table.4 Residual effect of preceding rice crop treatments on available nitrogen $\left(\mathrm{Kg} \mathrm{ha}^{-1}\right)$ in soil at different growth stages of wheat

\begin{tabular}{|l|c|c|c|c|c|c|c|c|}
\hline \multirow{2}{*}{ Treatments } & \multicolumn{2}{|c|}{ Tillering Stage } & \multicolumn{2}{c|}{ Jointing Stage } & \multicolumn{2}{c|}{ Heading Stage } & \multicolumn{2}{c|}{ Harvest } \\
\cline { 2 - 9 } & $\mathbf{2 0 1 1 - 1 2}$ & $\mathbf{2 0 1 2 - 1 3}$ & $\mathbf{2 0 1 1 - 1 2}$ & $\mathbf{2 0 1 2 - 1 3}$ & $\mathbf{2 0 1 1 - 1 2}$ & $\mathbf{2 0 1 2 - 1 3}$ & $\mathbf{2 0 1 1 - 1 2}$ & $\mathbf{2 0 1 2 - 1 3}$ \\
\hline $\mathrm{T}_{1}$ & 195.42 & 176.18 & 170.63 & 165.65 & 164.74 & 157.58 & 151.28 & 147.93 \\
\hline $\mathrm{T}_{2}$ & 209.14 & 210.32 & 203.79 & 204.82 & 193.49 & 194.69 & 203.75 & 199.35 \\
\hline $\mathrm{T}_{3}$ & 215.94 & 210.97 & 208.35 & 207.38 & 204.54 & 202.83 & 203.84 & 204.57 \\
\hline $\mathrm{T}_{4}$ & 216.48 & 213.45 & 212.44 & 210.62 & 209.24 & 206.42 & 205.48 & 208.74 \\
\hline $\mathrm{T}_{5}$ & 216.78 & 216.29 & 214.44 & 213.46 & 211.57 & 210.35 & 208.78 & 209.56 \\
\hline $\mathrm{T}_{6}$ & 218.65 & 219.36 & 218.56 & 216.27 & 214.84 & 213.47 & 209.68 & 211.24 \\
\hline $\mathrm{T}_{7}$ & 226.54 & 221.23 & 219.64 & 217.39 & 215.88 & 214.37 & 211.56 & 214.76 \\
\hline $\mathrm{T}_{8}$ & 226.36 & 222.74 & 225.93 & 223.75 & 219.45 & 219.57 & 215.72 & 215.73 \\
\hline $\mathrm{T}_{9}$ & 229.27 & 223.87 & 227.72 & 224.39 & 223.35 & 220.83 & 219.33 & 219.46 \\
\hline $\mathrm{T}_{10}$ & 231.85 & 227.45 & 229.58 & 226.53 & 226.24 & 222.78 & 221.93 & 223.56 \\
\hline $\mathrm{SE}(\mathrm{m})$ & 2.36 & 2.58 & 2.011 & 2.59 & 2.30 & 2.57 & 2.30 & 2.47 \\
\hline $\mathrm{CD}$ at $5 \%$ & 7.06 & 7.71 & 6.02 & 7.76 & 6.89 & 7.71 & 6.90 & 7.41 \\
\hline
\end{tabular}


Table.5 Residual effect of preceding rice crop treatments on available phosphorus $\left(\mathrm{Kg} \mathrm{ha}^{-1}\right)$ in soil at different growth stages of wheat

\begin{tabular}{|l|c|c|c|c|c|c|c|c|}
\hline \multirow{2}{*}{ Treatments } & \multicolumn{2}{|c|}{ Tillering Stage } & \multicolumn{2}{c|}{ Jointing Stage } & \multicolumn{2}{c|}{ Heading Stage } & \multicolumn{2}{c|}{ Harvest } \\
\cline { 2 - 9 } & $\mathbf{2 0 1 1 - 1 2}$ & $\mathbf{2 0 1 2 - 1 3}$ & $\mathbf{2 0 1 1 - 1 2}$ & $\mathbf{2 0 1 2 - 1 3}$ & $\mathbf{2 0 1 1 - 1 2}$ & $\mathbf{2 0 1 2 - 1 3}$ & $\mathbf{2 0 1 1 - 1 2}$ & $\mathbf{2 0 1 2 - 1 3}$ \\
\hline $\mathrm{T}_{1}$ & 12.43 & 14.74 & 12.20 & 13.58 & 10.73 & 12.85 & 10.25 & 11.29 \\
\hline $\mathrm{T}_{2}$ & 13.40 & 15.47 & 12.67 & 13.62 & 12.30 & 12.94 & 11.95 & 11.75 \\
\hline $\mathrm{T}_{3}$ & 14.78 & 16.67 & 13.37 & 13.84 & 12.14 & 12.97 & 11.65 & 11.88 \\
\hline $\mathrm{T}_{4}$ & 14.53 & 16.44 & 14.31 & 14.28 & 13.68 & 13.04 & 12.46 & 12.34 \\
\hline $\mathrm{T}_{5}$ & 16.56 & 16.37 & 14.49 & 14.84 & 14.07 & 14.44 & 13.43 & 12.73 \\
\hline $\mathrm{T}_{6}$ & 17.44 & 17.27 & 13.54 & 14.57 & 13.67 & 13.11 & 12.32 & 11.83 \\
\hline $\mathrm{T}_{7}$ & 14.38 & 16.25 & 13.17 & 13.55 & 12.71 & 13.24 & 11.76 & 11.64 \\
\hline $\mathrm{T}_{8}$ & 14.68 & 15.84 & 12.38 & 13.39 & 12.57 & 13.19 & 11.24 & 11.15 \\
\hline $\mathrm{T}_{9}$ & 16.34 & 16.85 & 13.67 & 13.87 & 12.88 & 13.31 & 11.85 & 11.68 \\
\hline $\mathrm{T}_{10}$ & 16.33 & 16.83 & 13.39 & 13.65 & 12.75 & 13.29 & 11.98 & 11.79 \\
\hline $\mathrm{SE}(\mathrm{m})$ & 2.18 & 2.11 & 2.10 & 1.97 & 1.94 & 2.09 & 2.13 & 1.98 \\
\hline $\mathrm{CD}$ at $5 \%$ & N.S. & N.S. & N.S. & N.S. & N.S. & N.S. & N.S. & N.S. \\
\hline
\end{tabular}

Table.6 Residual effect of preceding rice crop treatments on available potassium $\left(\mathrm{Kg} \mathrm{ha}^{-1}\right)$ in soil at different growth stages of wheat

\begin{tabular}{|l|c|c|c|c|c|c|c|c|}
\hline \multirow{2}{*}{ Treatments } & \multicolumn{2}{|c|}{ Tillering Stage } & \multicolumn{2}{c|}{ Jointing Stage } & \multicolumn{2}{c|}{ Heading Stage } & \multicolumn{2}{c|}{ Harvest } \\
\cline { 2 - 9 } & $\mathbf{2 0 1 1 - 1 2}$ & $\mathbf{2 0 1 2 - 1 3}$ & $\mathbf{2 0 1 1 - 1 2}$ & $\mathbf{2 0 1 2 - 1 3}$ & $\mathbf{2 0 1 1 - 1 2}$ & $\mathbf{2 0 1 2 - 1 3}$ & $\mathbf{2 0 1 1 - 1 2}$ & $\mathbf{2 0 1 2 - 1 3}$ \\
\hline $\mathrm{T}_{1}$ & 211.56 & 192.78 & 198.76 & 173.53 & 196.25 & 168.93 & 184.75 & 162.39 \\
\hline $\mathrm{T}_{2}$ & 230.28 & 233.73 & 228.10 & 232.75 & 225.38 & 229.46 & 224.84 & 224.64 \\
\hline $\mathrm{T}_{3}$ & 234.44 & 236.48 & 231.95 & 234.33 & 227.14 & 228.79 & 224.25 & 223.43 \\
\hline $\mathrm{T}_{4}$ & 235.76 & 249.93 & 232.85 & 243.22 & 228.27 & 233.83 & 225.43 & 227.85 \\
\hline $\mathrm{T}_{5}$ & 244.47 & 251.57 & 238.84 & 248.33 & 234.57 & 247.54 & 232.32 & 243.76 \\
\hline $\mathrm{T}_{6}$ & 245.50 & 251.83 & 239.65 & 247.74 & 235.39 & 244.55 & 233.85 & 240.26 \\
\hline $\mathrm{T}_{7}$ & 245.65 & 259.16 & 239.73 & 257.87 & 235.34 & 254.94 & 235.73 & 247.83 \\
\hline $\mathrm{T}_{8}$ & 245.72 & 261.26 & 239.83 & 259.57 & 236.42 & 256.32 & 233.79 & 250.85 \\
\hline $\mathrm{T}_{9}$ & 246.06 & 263.57 & 241.57 & 251.35 & 238.24 & 259.55 & 235.33 & 252.47 \\
\hline $\mathrm{T}_{10}$ & 247.08 & 264.04 & 244.60 & 262.83 & 241.86 & 260.77 & 239.14 & 254.35 \\
\hline $\mathrm{SE}(\mathrm{m})$ & 2.31 & 2.55 & 2.34 & 2.24 & 2.01 & 2.42 & 2.55 & 2.32 \\
\hline $\mathrm{CD}$ at $5 \%$ & 6.92 & 7.64 & 7.02 & 6.70 & 6.02 & 7.25 & 7.63 & 6.96 \\
\hline
\end{tabular}


Table.7 Residual effect of preceding rice crop treatments on organic carbon (\%) of soil in different growth stages of wheat crop 2011-12 to 2012-13

\begin{tabular}{|l|c|c|c|c|c|c|c|c|}
\hline \multirow{2}{*}{ Treatments } & \multicolumn{2}{|c|}{ Tillering Stage } & \multicolumn{2}{c|}{ Jointing Stage } & \multicolumn{2}{c|}{ Heading Stage } & \multicolumn{2}{c|}{ Harvest } \\
\cline { 2 - 9 } & $\mathbf{2 0 1 1 - 1 2}$ & $\mathbf{2 0 1 2 - 1 3}$ & $\mathbf{2 0 1 1 - 1 2}$ & $\mathbf{2 0 1 2 - 1 3}$ & $\mathbf{2 0 1 1 - 1 2}$ & $\mathbf{2 0 1 2 - 1 3}$ & $\mathbf{2 0 1 1 - 1 2}$ & $\mathbf{2 0 1 2 - 1 3}$ \\
\hline $\mathrm{T}_{1}$ & 0.388 & 0.382 & 0.385 & 0.377 & 0.383 & 0.373 & 0.378 & 0.369 \\
\hline $\mathrm{T}_{2}$ & 0.459 & 0.463 & 0.456 & 0.458 & 0.454 & 0.456 & 0.452 & 0.454 \\
\hline $\mathrm{T}_{3}$ & 0.468 & 0.475 & 0.465 & 0.473 & 0.463 & 0.469 & 0.460 & 0.464 \\
\hline $\mathrm{T}_{4}$ & 0.462 & 0.475 & 0.460 & 0.473 & 0.458 & 0.471 & 0.456 & 0.469 \\
\hline $\mathrm{T}_{5}$ & 0.471 & 0.478 & 0.469 & 0.476 & 0.466 & 0.474 & 0.464 & 0.472 \\
\hline $\mathrm{T}_{6}$ & 0.468 & 0.474 & 0.465 & 0.469 & 0.463 & 0.467 & 0.460 & 0.465 \\
\hline $\mathrm{T}_{7}$ & 0.465 & 0.460 & 0.463 & 0.458 & 0.460 & 0.456 & 0.459 & 0.454 \\
\hline $\mathrm{T}_{8}$ & 0.457 & 0.459 & 0.456 & 0.464 & 0.453 & 0.463 & 0.452 & 0.460 \\
\hline $\mathrm{T}_{9}$ & 0.469 & 0.463 & 0.465 & 0.461 & 0.463 & 0.460 & 0.461 & 0.458 \\
\hline $\mathrm{T}_{10}$ & 0.478 & 0.483 & 0.476 & 0.481 & 0.474 & 0.478 & 0.472 & 0.476 \\
\hline $\mathrm{SE}(\mathrm{m})$ & 0.024 & 0.026 & 0.025 & 0.025 & 0.026 & 0.024 & 0.026 & 0.027 \\
\hline $\mathrm{CD}$ at $5 \%$ & N.S. & N.S. & N.S. & N.S. & N.S. & N.S. & N.S. & N.S. \\
\hline
\end{tabular}

Table.8 Residual effect of preceding rice crop treatments on $\mathrm{pH}$ of soil at different growth stages of Wheat 2011-12 to 2012-13

\begin{tabular}{|l|c|c|c|c|c|c|c|c|}
\hline \multirow{2}{*}{ Treatments } & \multicolumn{2}{|l|}{ Tillering Stage } & \multicolumn{2}{l}{ Jointing Stage } & \multicolumn{2}{l}{ Heading Stage } & \multicolumn{2}{l|}{ Harvest } \\
\cline { 2 - 9 } & $\mathbf{2 0 1 1 - 1 2}$ & $\mathbf{2 0 1 2 - 1 3}$ & $\mathbf{2 0 1 1 - 1 2}$ & $\mathbf{2 0 1 2 - 1 3}$ & $\mathbf{2 0 1 1 - 1 2}$ & $\mathbf{2 0 1 2 - 1 3}$ & $\mathbf{2 0 1 1 - 1 2}$ & $\mathbf{2 0 1 2 - 1 3}$ \\
\hline $\mathrm{T}_{1}$ & 8.15 & 8.09 & 8.03 & 7.97 & 7.91 & 7.85 & 7.79 & 7.73 \\
\hline $\mathrm{T}_{2}$ & 7.19 & 7.88 & 7.75 & 7.72 & 7.59 & 7.56 & 7.43 & 7.40 \\
\hline $\mathrm{T}_{3}$ & 7.82 & 8.04 & 7.68 & 7.97 & 7.54 & 7.93 & 7.40 & 7.87 \\
\hline $\mathrm{T}_{4}$ & 7.79 & 8.02 & 7.66 & 7.95 & 7.53 & 7.76 & 7.40 & 7.66 \\
\hline $\mathrm{T}_{5}$ & 7.74 & 7.97 & 7.61 & 7.54 & 7.48 & 7.41 & 7.35 & 7.28 \\
\hline $\mathrm{T}_{6}$ & 7.75 & 7.69 & 7.62 & 7.56 & 7.49 & 7.43 & 7.36 & 7.30 \\
\hline $\mathrm{T}_{7}$ & 7.69 & 7.72 & 7.64 & 7.62 & 7.58 & 7.48 & 7.52 & 7.34 \\
\hline $\mathrm{T}_{8}$ & 7.66 & 7.98 & 7.61 & 7.91 & 7.55 & 7.87 & 7.50 & 7.82 \\
\hline $\mathrm{T}_{9}$ & 7.65 & 7.96 & 7.60 & 7.88 & 7.53 & 7.83 & 7.49 & 7.77 \\
\hline $\mathrm{T}_{10}$ & 7.68 & 7.67 & 7.63 & 7.59 & 7.57 & 7.46 & 7.53 & 7.36 \\
\hline $\mathrm{SE}(\mathrm{m})$ & 0.55 & 0.51 & 0.23 & 0.18 & 0.24 & 0.16 & 0.26 & 0.19 \\
\hline $\mathrm{CD}$ at 5\% & N.S. & N.S. & N.S. & N.S. & N.S. & N.S. & N.S. & N.S. \\
\hline
\end{tabular}


Table.9 Residual effect of preceding rice crop treatments on EC $\left(\mathrm{dSm}^{-1}\right)$ of soil at different growth stages of wheat 2011-12 to 2012-13

\begin{tabular}{|l|l|l|l|l|l|l|l|l|}
\hline \multirow{2}{*}{ Treatments } & \multicolumn{2}{|c|}{ Tillering Stage } & \multicolumn{2}{c|}{ Jointing Stage } & \multicolumn{2}{c|}{ Heading Stage } & \multicolumn{2}{c|}{ Harvest } \\
\cline { 2 - 9 } & $\mathbf{2 0 1 1 - 1 2}$ & $\mathbf{2 0 1 2 - 1 3}$ & $\mathbf{2 0 1 1 - 1 2}$ & $\mathbf{2 0 1 2 - 1 3}$ & $\mathbf{2 0 1 1 - 1 2}$ & $\mathbf{2 0 1 2 - 1 3}$ & $\mathbf{2 0 1 1 - 1 2}$ & $\mathbf{2 0 1 2 - 1 3}$ \\
\hline $\mathrm{T}_{1}$ & 0.28 & 0.25 & 0.28 & 0.25 & 0.24 & 0.23 & 0.31 & 0.29 \\
\hline $\mathrm{T}_{2}$ & 0.22 & 0.22 & 0.24 & 0.21 & 0.22 & 0.20 & 0.25 & 0.24 \\
\hline $\mathrm{T}_{3}$ & 0.21 & 0.23 & 0.22 & 0.20 & 0.23 & 0.20 & 0.23 & 0.25 \\
\hline $\mathrm{T}_{4}$ & 0.23 & 0.22 & 0.25 & 0.23 & 0.21 & 0.19 & 0.26 & 0.24 \\
\hline $\mathrm{T}_{5}$ & 0.21 & 0.24 & 0.21 & 0.22 & 0.21 & 0.21 & 0.25 & 0.26 \\
\hline $\mathrm{T}_{6}$ & 0.23 & 0.22 & 0.24 & 0.23 & 0.22 & 0.20 & 0.24 & 0.25 \\
\hline $\mathrm{T}_{7}$ & 0.24 & 0.23 & 0.25 & 0.24 & 0.24 & 0.22 & 0.25 & 0.27 \\
\hline $\mathrm{T}_{8}$ & 0.26 & 0.25 & 0.24 & 0.24 & 0.22 & 0.23 & 0.28 & 0.26 \\
\hline $\mathrm{T}_{9}$ & 0.26 & 0.24 & 0.26 & 0.25 & 0.22 & 0.24 & 0.29 & 0.25 \\
\hline $\mathrm{T}_{10}$ & 0.25 & 0.23 & 0.24 & 0.24 & 0.23 & 0.22 & 0.27 & 0.24 \\
\hline $\mathrm{SE}(\mathrm{m})$ & 0.01 & 0.01 & 0.01 & 0.01 & 0.01 & 0.01 & 0.01 & 0.01 \\
\hline $\mathrm{CD}$ at $5 \%$ & 0.02 & 0.02 & 0.02 & 0.02 & 0.02 & 0.02 & 0.02 & 0.04 \\
\hline
\end{tabular}

Maximum biomass production than the other treatments was found in case of $\mathrm{T}_{10}$ during both the years. Treatments consisting application of 4 ton vermicompost during preceding rice crop did not differ significantly in respect of biomass production.

All the treatments consisting 4 ton vermicompost to rice and $100 \%$ NPK to wheat produced significantly higher grain and straw yield of wheat than $\mathrm{T}_{2}$ where $100 \%$ NPK was applied to rice as well as wheat.

This significant variation in crop yield may be supposed due to improved physical environment of soil due to residual effect of vermicompost that had resulted in better plant growth and yield attributing characters with residual effect nutrients availability were also higher (Aruna and Shaik, 2005). Application of farmyard manure at 10 tons ha $^{-1}$ contributes $30-60 \mathrm{~kg} \mathrm{~N} \mathrm{ha}^{-1}$ in rice besides leaving a significant residual effect on succeeding crop (Sharma, 1995). Bhandari et al., (1992) also reported the residual effect of organic sources applied to rice on succeeding wheat. Significant residual effect on wheat crop was also reported by Rajput and Warsi (1992).

\section{$\mathrm{N}, \mathbf{P}$, and $\mathrm{K}$ availability}

At tillering stage available soil $\mathrm{N}\left(\mathrm{kg} \mathrm{ha}^{-1}\right)$ varied from 195.42 to 231.85 and 176.18 to 227.45 during 2011-12 and 2012-13, respectively (Table 4). In most cases the residual effect of vermicompost application to rice on available soil $\mathrm{N}$ at this stage of wheat was significant during both the years. Availability of $\mathrm{N}$ in $\mathrm{T}_{2}$ was found significantly lower than the treatments where vermicompost was applied during Kharif season. Available soil $\mathrm{N}$ at jointing stage declined from the value recorded at tillering during both the years. Available $\mathrm{N}$ in soil at this stage varied from 170.63 to 229.58 and 165.65 to $226.53 \mathrm{~kg} \mathrm{ha}^{-1}$ during 2011-12 and 2012-13, respectively. Most of the Treatments having vermicompost application during rice season exhibited their significant residual effect over $\mathrm{T}_{2}$ in respect of available soil $\mathrm{N}$ during both the years. Availability of soil N was slightly higher with the delayed application of vermicompost to rice. The available $\mathrm{N}$ in soil at heading stage varied from 164.74 to 226.24 and 157.58 to 222.78 $\mathrm{kg} \mathrm{ha}{ }^{-1}$ during 2011-12 and 2012-13, respectively. All the treatments consisting 
application of vermicompost during rice crop exhibited a significant effect over $\mathrm{T}_{2}$. Delayed application of either 2 or 4 ton vermicompost in rice season showed slightly higher $\mathrm{N}$ availability. At harvesting available $\mathrm{N}$ in soil differ significantly under different treatments and varied from 151.28 to 221.93 and 147.93 to $223.56 \mathrm{~kg} \mathrm{ha}^{-1}$ during 2011-12 and 2012-13 respectively.

At tillering stage available $\mathrm{P}$ in soil varied from 12.43 to 17.44 and 14.74 to $17.27 \mathrm{~kg}$ ha 1 during 2011-12 and 2012-13 respectively (Table 5). Minimum available $\mathrm{P}$ in soil was found in the control plot $\left(\mathrm{T}_{1}\right)$, while maximum available $\mathrm{P}$ in the plot fertilized with $75 \% \mathrm{~N}, 100 \% \mathrm{P}$ and $\mathrm{K}+$ vermicompost @ 2 ton $\mathrm{ha}^{-1}$ at flowering stage of rice and $100 \%$ NPK to wheat $\left(\mathrm{T}_{6}\right)$ during both the years. At jointing stage available $\mathrm{P}$ in soil decline from the previous content in all the treatments and ranged from 12.20 to 14.49 and 13.58 to $14.84 \mathrm{~kg} \mathrm{ha}^{-1}$ during 2011-12 and 2012-13 respectively. Minimum availability was found in $\mathrm{T}_{1}$ while maximum in $\mathrm{T}_{5}$. The available $\mathrm{P}$ in soil at heading stage further decreased in all the treatments and varied from 10.73 to 14.07 and 12.85 to 14.44 $\mathrm{kg} \mathrm{ha}{ }^{-1}$ during 2011-12 and 2012-13, respectively. At harvest $\mathrm{P}$ in soil varied from 10.25 to 13.43 and 11.29 to $12.73 \mathrm{~kg} \mathrm{ha}^{-1}$ during 2011-12 and 2012-13, respectively.

Soil available $\mathrm{K}$ at jointing stage declined from the value recorded at tillering during both the years under different treatments. Available $\mathrm{K}$ in soil at this stage varied from 198.76 to 244.60 and 173.53 to 262.83 (kg ha $\left.{ }^{1}\right)$ during 2011-12 and 2012-13, respectively (Table 6). The available $\mathrm{K}$ in soil at heading stage varied from 196.245 to 241.863 and 168.93 to $260.77 \mathrm{~kg} \mathrm{ha}^{-1}$ during 2011-12 and 2012-13 respectively. At harvesting available soil $\mathrm{K}$ varied from 184.75 to 239.14 and 162.39 to $254.35 \mathrm{~kg} \mathrm{ha}^{-1}$ during 2011-12 and 2012-13, respectively. Maximum available K at this stage was found in $\mathrm{T}_{10}$ during both the years. At harvest it was also noticed that availability of potassium was significantly lower with the basal application of vermicompost than at flowering stage in previous rice crop. Minimum and significantly lower available soil $\mathrm{K}$ than the rest of the treatments during both the years was found in $\mathrm{T}_{1}$ control.

In comparison to $\mathrm{T}_{2}$ of $\mathrm{N}$ and $\mathrm{K}$ in soil at different growth stages was significantly higher in the treatments where vermicompost was applied. Since the added organic sources did not mineralized completely within the season of application, some portion is left undecomposed or un-mineralized this portion benefit the succeeding crop through the supplementation of plant nutrients and improvement in soil physical environment (Santhi et al., 1999 and Kamla Kanwar, 2002). Substantial improvement in soil fertility due to residual effect was also reported by Aruna and Shaik, 2005. Residual effect of different treatments applied to soyabean on succeeding wheat was also reported by Kundu et al., 2006. Phosphorous availability did not differ significantly due to residual effect although a significant difference was expected.

\section{Organic carbon (\%), pH and Electrical Conductivity $\left(\mathrm{dSm}^{-1}\right)$}

At tillering stage the organic carbon percent varied from 0.388 to 0.478 and 0.382 to 0.483 during 2011-12 and 2012-13, respectively (Table 7). The highest organic carbon percent 0.478 and 0.483 percent were recorded in $\mathrm{T}_{\mathbf{1 0}}$ during 2011-12 and 2012-13, respectively, where along with $50 \% \mathrm{~N}, 100 \% \mathrm{P}$ and $\mathrm{K}, 4$ ton vermicompost was applied at flowering stage of preceding rice crop. Further with the advancement in crop growth percent organic carbon declined in all the treatments during both the years. At jointing, heading and 
harvest percent organic carbon varied from 0.385 to $0.476,0.383$ to 0.474 and 0.378 to 0.472 , respectively during 2011-12 while 0.377 to $0.481,0.373$ to 0.478 and 0.369 to 0.476 , respectively during 2012-13.

At tillering stage the soil $\mathrm{pH}$ varied from 7.19 to 8.15 and 7.67 to 8.09 during 2011-12 and 2012-13, respectively (Table 8). The maximum $\mathrm{pH} 8.15$ and 8.09 during 2011-12 and 2012-13, respectively, was recorded in $T_{1}$ where no fertilizer was applied. The minimum soil $\mathrm{pH} 7.65$ and 7.67 during 2011-12 and 2012-13, respectively was found with the delayed application of 4 ton vermicompost to rice crop. Soil $\mathrm{pH}$ further declined with the advancement in crop growth. In all the cases lower soil $\mathrm{pH}$ was recorded in the treatments receiving vermicompost application during the rice crop cultivation.

At tillering stage electrical conductivity $(\mathrm{dSm}$ ${ }^{1}$ ) of soil varied from 0.21 to 0.28 and 0.22 to $0.25 \mathrm{dSm}^{-1}$ during 2011-2012 and 2012-2013, respectively (Table 9). The highest electrical conductivity at this stage was found in $T_{1}$ during both the years. Electrical conductivity of soil at jointing stage varied from 0.21 to 0.28 and 0.20 to $0.25\left(\mathrm{dSm}^{-1}\right)$ during 2012 and 2013, respectively. At this stage maximum electrical conductivity of soil 0.28 and $0.25\left(\mathrm{dSm}^{-1}\right)$ during 2011-12 and 2012-13 respectively, was also found in $\mathrm{T}_{1}$. Electrical conductivity ranged from 0.21 to 0.24 and 0.23 to $0.31 \mathrm{dSm}^{-1}$ at heading stage and wheat harvest, respectively, during 2011-12 while 0.19 to 0.23 and 0.24 to $0.29 \mathrm{dSm}^{-1}$ respectively during 2012-13. Although slightly higher values were recorded with the application of higher dose of vermicompost but a particular trend of treatments effect at different growth stages was not observed.

Residual effect of applied vermicompost to rice on organic carbon content of soil during the growth of wheat was non-significant.
Similarly the residual effect on soil $\mathrm{pH}$ was also non-significant. Soil $\mathrm{pH}$ and organic carbon percent in soil declined with the advancement of wheat crop growth. With the decomposition of organic matter different organic acids are produced which are supposed to reduce the soil $\mathrm{pH}$ (Rajput and Warsi, 1992). Electrical conductivity was higher due to residual effect. It may be explained due to release of different minerals by the organic matter decomposition that will enrich the salt concentration of soil solution.

\section{References}

Aruna, E. and Shaik, M. 2005. Influence of conjuctive use of organic and inorganic source of nutrients in rice (Oryza sativa) on crop growth, yield components, yield and soil fertility in rice sunflower (Helianthus annuus) sequence. Indian Journal of Agronomy. 50 (4): 265-268.

Bhandari, A.L., Sood, A., Sharma, K.N. and Rana, D.S. 1992. Integrated Nutrient Management in Rice-Wheat System. $J$. Indian Soc. Soil Sci. 40: 742-747.

Das, P.K., Jen, M.K. and Sahoo, K.C. 2002. Effect of integrated application of vermicompost and chemical fertilizer on growth and yield of paddy in red soil of South Eastern Ghat Zone of Orissa. Environ and Ecology. 20(1): 13-15.

Dussere, C. 1992. On the effect of earthworm on the chemical condition of soil. Landbow J. B. Schwiez. 16:75-78.

Gomez, A.K. and Gomez. A.A. 1984. Statistical procedures for Agricultural Research Second Edition. John Wiley and Sons, New York. U.S.A.

Jackson, M.L. 1967. Soil chemical analysis prentice hall of India private limited. New Delhi.

Kamla, K. 2002. Recycling of organic wastes by vermicomposting and its utilization in agriculture in Himachal Pradesh,. Indian. J. Soil. Sci. 24(2): 12-25. 
Kundu, S., Bhattacharyyal, R. Prakash, V., Gupta, H.S., Pathak, H. and Ladha, K. 2006. Effects of organic and inorganic sources of nutrients on grain yield trends of rainfed soybean-wheat system and nutrient status in a sandy loam soil. J. Indian, Soc. Soil. Sci. 52(1): 45-47.

Olsen, S.R., Cole, C.V., Watanable, F.S. and Dean, L.A. 1954. Estimation of available phosphorus in soil by extraction with sodium bicarbonate. Circular 939 United States Department of Agriculture. pp 19.

Rajput, A.I. and Warsi, A.S. 1992. Effect of nitrogen organic manure on rice (oryza sativa) yield and residual effect on wheat (Thriticum aestivum) crop. Indian. J. Agron. 37(4): 716-720.

Rao, K.R., Rao, P.A. and Rao, K.t. 2000. Influence of organic manure and fertilizers on the incidence of groundnut leafminer. Approacrema., Modicella Dev Annals of Plant Protection Sci. 9(1): 12-15.

Richards, L. A. 1954. Diagnosis and improvement of saline-alkali soils. USDA Handbook No. 60 U.S.
Department of Agriculture. Washington, D.C. (USA).

Robinson. C. H. Inceson, P. Pierce, T.G. and Rowland, A.P. 1992. Nitrogen mobilization by earthworm in limed peat soils under Picea sitchensis, $J$. Applied Ecol. 29: 226-237.

Santhi, F. Velusamy, M.S. Murugappan, V.M. and Selvi, D. 1999. Effect of inorganic fertilizers and fertilizer manure combination on soil physico-chemical properties and dynamics of microbial biomass in an inceptisol. J. Indian. Soc. Soil. Sci. 47(3): 479-482.

Sharma, A.R. Fertilizer Use in Rice and Rice Based Cropping System Fert. News 1995, 40(5): 29-41.

Singh, R.K., Athokpam, H.S. and Zaliana, C., Singh, N.G. 2005. Integrated management of Azolla, vermicompost and urea on yield of and nutrient uptake by rice and soil fertility. J. Indian, Soc. Soil. Sci. 53(1): 107-110.

Vasanthi, D. and Kumarswamy, K. 2000. Efficacy of vermicompost to improve soil fertility and rice yield. Indian $J$. Soc. Soil. Sci. 47(2): 268-272.

\section{How to cite this article:}

Amit Kumar, B.P. Dhyani, Ashish Rai and Vipin Kumar. 2017. Residual Effect of Applied Vermicompost and NPK to Rice on Growth and Yield of Succeeding Wheat and Chemical Properties of Soil. Int.J.Curr.Microbiol.App.Sci. 6(11): 1087-1098. doi: https://doi.org/10.20546/ijcmas.2017.611.128 